\title{
Algunos pensamientos sobre la tormenta que se avecina en las redes sociales, el medio ambientey la necesidad de un diálogo transformadory democrático
}

Sección ESTUDI0S

RECIBIDO: $11 / 10 / 2021$

APROBADO: $12 / 11 / 2021$

PUBLICADO ONLINE: 30/12/2021

\author{
Paul R. Carr \\ Université du Québec en Outaouais (UQ0) \\ prcarr@gmail.com \\ https://0rcid.org/0000-0002-3841-0086
}

\section{RESUMEN}

Este texto presenta algunos argumentos que vinculan las redes sociales, el medio ambiente y la necesidad de un diálogo transformador, en el contexto de marcos teóricos superpuestos de la democracia. Sostengo que la manipulación de los medios y las noticias falsas no son un fenómeno nuevo, pero que el contexto de las redes sociales ha complicado la diseminación y sofisticación de los procesos hegemónicos que cubren todos los rincones del mundo. Las conspiraciones que sustentan el movimiento anti-vax, que impugnan la derrota del presidente Trump, la xenofobia y muchas otras proporcionan algunos contextos de cómo el medio ambiente es y puede ser distorsionado a través de mensajes virales, mitos urbanos, comentarios, videos, textos y otros medios dentro de un torrente de ideología anti-ciencia. El texto concluye con algunas reflexiones sobre obstáculos, implicaciones y consecuencias particulares, así como innovaciones que pueden ser útiles para hacer avanzar un diálogo significativo en relación con la agencia, la acción y el activismo ambiental.

PALABRAS CLAVE: redes sociales, noticias falsas, medio ambiente, diálogo, democracia

Some thoughts on the gathering storm over social media, the environment and the need for transformative dialogue and democracy

\footnotetext{
ABSTRACT

This text presents arguments linking together social media, the environment and the need for transformative dialogue. Within the backdrop of overlapping frameworks of democracy, I argue that media manipulation and fake news are not a new phenomenon but that the social media context has complexified the dissemination and sophistication of hegemonic processes that cover all corners of the Globe. Conspiracies underpinning the anti-vax movement, contesting the defeat of President Trump, xenophobia and many others provide some context for how the environment is and can be

(c) Los autores. Este artículo es publicado por la Revista de Sociología de la Facultad de Ciencias Sociales, Universidad Nacional Mayor de San Marcos. Este es un artículo de acceso abierto, distribuido bajo los términos de la licencia Creative Commons Atribución 4.0 Internacional (CC BY 4.0) [https://creativecommons.org/licenses/by/4.0/deed.es] que permite el uso, distribución y reproducción en cualquier medio, siempre que la obra original sea debidamente citada de su fuente original.
} 
distorted through viral, urban myth messages, comments, videos, texts and other media within a torrent of anti-science ideology. The text concludes with some thoughts on particular obstacles, implications and consequences as well as innovations that may be helpful in moving meaningful dialogue forward in relation to environmental agency, action and activism.

KEYWORDS: social media, fake news, environment, dialogue, democracy

\section{Introducción ${ }^{1}$}

L

a pandemia de COVID ha sido una catástrofe para la salud mundial y, por las desigualdades sociales, muchas partes del mundo han sido excluidas de la lotería de vacunas (Carr, 2020). Sin embargo, la crisis mundial en torno al rápido contagio del virus potencialmente letal y de largo alcance, que no es el primero de este tipo, pero la única preocupación mundial ahora es que corre el riesgo de alterar significativamente el destino humano. La situación ambiental que precedió al COVID no ha desaparecido, e incluso se podría argumentar que la emergencia y la urgencia están ahora (casi) más allá del punto de no hay vuelta atrás. De hecho, la imagen proverbial de la medianoche de 5 minutos menos ya no es una exageración. Las innumerables manifestaciones climáticas visibles e invisibles que afectan a la tierra, al agua, al aire, a los seres humanos y a todas las especies, y a todo lo que se encuentra en el medio, ahora se pueden documentar y experimentar (Carr, 2021).

Una pieza fundamental de la ecuación de como entendemos el cambio / impacto ambiental junto al marco político y económico que sustenta la toma de decisiones, la movilización y la acción que se resume en la forma como las personas interactúan, se organizan, participan y se comprometen entre sí (Blue, 2015). Por lo tanto, las redes sociales se vuelven centrales en este debate, dado a su potencial que brinda una comunicación democrática y generalizada, pero que también está imbuido de innumerables problemas y consecuencias (Keating y Melis, 2017; Wagner y Payne, 2017). El problema de las "fake news" es importante, porque cómo la gente está informada, cómo consumen información, cómo dialogan con otros y cómo construyen conocimiento juntos puede ser determinante en relación con la acción significativa y el cambio relacionado con el medio ambiente (Blythe, Daigle y Baird, 2019; MacKenzie y Bhatt, 2018).

Este texto conceptual presenta algunos argumentos que vinculan las redes sociales, el medio ambiente y la necesidad de un diálogo transformador, en el contexto de marcos teóricos superpuestos de la democracia (Carr, 2020). Utilizo

1 Agradezco mucho a mi estudiante de doctorado y asistente de investigación de la Cátedra UNESCO DCMÉT, Ivette Doizi, por su apoyo en la traducción de este texto. 
las redes sociales como un sitio de investigación y como una metodología para comprender, problematizar y examinar las tendencias, consecuencias, impactos y pertinencia de las comunicaciones en línea. Sostengo que la manipulación de los medios y las noticias falsas no son un fenómeno nuevo, pero que el contexto de las redes sociales ha complicado la diseminación y sofisticación de los procesos hegemónicos que cubren todos los rincones del mundo (Carr, Cuervo y Daros, 2019; Carr, Daros, Cuervo y Thésée, 2020). Las conspiraciones que sustentan el movimiento anti-vax, que impugnan la derrota del presidente Trump, la xenofobia y muchas otras proporcionan algunos contextos de cómo el medio ambiente es y puede ser distorsionado a través de mensajes virales, mitos urbanos, comentarios, videos, textos y otros medios dentro de un torrente de ideología anti-ciencia (De Coninck et al., 2021). El texto concluye con algunas reflexiones sobre obstáculos, implicaciones y consecuencias particulares, así como innovaciones que pueden ser útiles para hacer avanzar un diálogo significativo en relación con la agencia, la acción y el activismo ambiental.

\section{La democracia y el contexto de las redes sociales}

La democracia es contestada por una serie de razones, y lo que se considera democracia normativa, que prioriza el voto, las elecciones, los partidos políticos y las instituciones principales, está cada vez más marginada como el único o principal motor de la vida política (que se puede etiquetar como Democracia 1.0 (Carr, 2020, 2021). Democracia 2.0 (con una apertura a la justicia social, diversas formas de organización / movilización, una explosión de potencial creatividad, una crítica al neoliberalismo que al mismo tiempo se ve limitado por él, y los medios más democratizados). Esta es mucho más desordenada que la Democracia 1.0 y es más complicada por la infiltración de la Democracia 3.0 (con predominio a la vigilancia, los algoritmos, la inteligencia artificial y en un estrecho marco del mundo). Las redes sociales han alterado de muchas maneras la forma en que consideramos el mundo, así como nuestra capacidad para cambiar e involucrarnos con él (Carr, Cuervo y Daros, 2019; Carr, Daros, Cuervo y Thésée, 2020).

La noción binaria, "bipartidista" de comprender hechos y realidades altamente complejas, creo que todavía el mundo de la Democracia 1.0 está en conflicto con los entornos de la Democracia 2.0. Todo ello reforzado por las restricciones algorítmicas basadas en la vigilancia que presenta la Democracia 3.0. Más allá de la democracia normativa, dominante y hegemónica, centrada en las elecciones, los partidos polí- 
ticos, las instituciones, el mantra de "la libertad"y "el gobierno para todos", que ha sido la columna vertebral de la Democracia 1.0, ahora, se enfrenta a los movimientos sociales, las redes sociales y a un debate más amplio, inclusivo y deliberativo y a la justicia social, y la problematización de la distribución y el reparto del ingreso, que están instalados en la Democracia 2.0. Me preocupa particularmente aquí el significado y el papel de la educación, y cómo puede contribuir a conceptualizar, desarrollar, cultivar, construir y elaborar una democracia más decente, significativa, robusta y críticamente comprometida (Carr, Hoechsmann, y Thésée, 2018; Carr y Thésée, 2019; Hoechsmann, Carr y Thésée, 2021).

Entonces me pregunto, dentro del contexto virtual que está en evolución, ¿qué es lo que se convierte en noticia, realmente en viral, y cómo se convierte en algo más que un simple clic. el entretenimiento algorítmico, el rebote en las cámaras de eco, el intercambio tibio y una plataforma para los trolls (persona que perturba el buen vivir)? ¿Se trata de números, de la cantidad de clics, vistas, acciones y lecturas, o de algo más sustantivo? Al mismo tiempo, ¿cuáles son las verdaderas dimensiones de la cuestión? ¿Quién lo enmarca, cómo y por qué, y con qué fin? ¿Qué se omite, se minimiza, se ofusca, cómo y por qué? (Hoechsmann, Carr y Thésée, 2021).

Hay muchas trampas, debilidades y preocupaciones problemáticas en cuanto a lo que son los "asuntos". Aunque no todos los asuntos emanan o conciernen directamente a los EE.UU. - y no queremos descartar aquí la hegemonía occidental, las maquinaciones geopolíticas, los legados coloniales, el hiperneoliberalismo y el papel de países como Canadá, etc.- l la presencia, la amplitud y la conexión para y con los Estados Unidos es palpable, así como la importante interacción entre la democracia 1.0 y la democracia 2.0. Todas las cuestiones no son simplemente un problema hegemónico de EE.UU., pero creo que vale la pena establecer las conexiones con las élites, la hegemonía, las diferencias de poder y el marco de los medios de comunicación.

\section{Las redes sociales y diversas formas de diálogo}

Por otro lado, con colegas (Carr, Daros, Cuervo, y Thésée, 2020), describimos algunos de los componentes, procesos y preocupaciones que se superponen y que ayudan a enmarcar el contexto de las redes sociales, las noticias falsas y la participación ciudadana en el contexto de estas formas divergentes y superpuestas de la democracia. Pareciera que hoy en día todo el mundo está conectado de alguna manera a las redes sociales, incluso si uno no tiene una cuenta en una o varias plataformas 
que están impregnando, enlazando y asfixiando el paisaje sociocultural (Keating y Melis, 2017). ${ }^{2}$

Hay redes sociales para una variedad incalculable de intercambio y recopilación de información. Los sustantivos se han convertido en verbos como "youtubbing", "blogging", "vlogging", "googling", "facebooking", etc. El alcance es significativo, y las huellas digitales (y las huellas de los pies) son igualmente proporcionales (Sun, Wang, Shen y Zhang, 2015). Uno puede hacer una búsqueda de un par de zapatos en Amazon.com y, por arte de magia, aparecerán anuncios de zapatos en el feed personal de Facebook inmediatamente después. Los algoritmos programan cada vez más lo que vemos, y alinean al menos parte de nuestra atención en "cosas", a falta de una palabra mejor, en las que de otro modo no estaríamos interesados. Los algoritmos programan cada vez más lo que vemos, y alinean al menos parte de nuestra atención en "cosas", en las que de otro modo no estaríamos interesados, por la falta de una palabra mejor. Esta vigilancia, usurpación y recopilación de datos quedó significativamente expuesta en 2018, destacando Facebook por un año especialmente negativo que marcó un antes y un después (Sutton, 2018; Wong y Morris, 2018). Entre la letanía de acontecimientos, problemas y fenómenos que han asolado a Facebook, que evidentemente no se limitan a esta única prominente red social, fueron las siguientes afirmaciones, hallazgos y pruebas, entre otras cuestiones: algoritmos conectados a los "efectos negativos para el tráfico de referencia", anuncios no regulados que subrayaron la investigación de Mueller que tiene, como foco, en gran parte, la participación rusa en las elecciones presidenciales de Estados Unidos de 2016. Además, del escándalo de Cambridge Analytica que "obtuvo los datos de decenas de millones de usuarios de Facebook sin su conocimiento o consentimiento para ayudar a construir una poderosa herramienta de influencia política". Así como también, problemas de privacidad y seguridad, "acuerdos especiales de intercambio de datos con fabricantes de tecnología como Amazon, Apple y Samsung", hackeo de cuentas y problemas de regulación (Sutton, 2018).

Así, todos nos vemos afectados por los tentáculos explícitos e implícitos de los medios de comunicación social, y por la forma en que nuestras huellas digitales y acciones nos rastrean y siguen (Sun, Wang, Shen y Zhang, 2015). El impacto potencial es casi infinito en la forma en que podemos ser moldeados por el marketing dirigido, las noticias, la información y las tendencias y de otras maneras. Es importante destacar que la forma en que pensamos sobre el medio ambiente se ve muy afectada por los debates en línea.

2 Todas las citas en este texto fueron traducidas por el autor y su asistente de investigación del inglés original. 
La preocupación por la información engañosa, aunque no es nueva, es significativa en el control del debate. Según la Universidad de West Florida (2019), citando el trabajo de Melissa Zimdars del Merrmack College, las fake news pueden considerarse dentro del siguiente marco:

CATEGORÍA 1: Sitios web falsos o regularmente engañosos que se comparten en Facebook y las redes sociales. Algunos de estos sitios web utilizan titulares distorsionados e información descontextualizada o dudosa para generar "likes", "shares" y beneficios.

CATEGORÍA 2: Sitios web que pueden difundir información engañosa y/o potencialmente poco fiable.

CATEGORÍA 3: Sitios web que a veces utilizan titulares y descripciones en las redes sociales.

CATEGORÍA 4: Sitios de sátira/comedia, que pueden ofrecer importantes comentarios críticos sobre la política y la sociedad, pero tienen el potencial de ser compartidos como noticias reales/literales.

Enfatizando en que las noticias falsas rara vez se empaquetan limpiamente dentro de una sola categoría, la autora (Universidad de West Florida, 2019) advierte que el engaño necesita ser interrogado en varios momentos mientras se ven los mensajes de los medios de comunicación. Con la avalancha de cuentas falsas, usuarios falsos (bots) y algoritmos falsos (o manipulados), el terreno es fértil para las noticias falsas, sobre todo si los usuarios, consumidores y ciudadanos están condicionados a no cuestionar ni verificar lo que les llega, son reacios a descreer de las autoridades que suponen que no trabajan en su beneficio, son ignorantes, están desinteresados o están envueltos en ciclos informativos turbulentos con información compleja, matizada y voluminosa. De esta manera, son incapaces de descifrar las diversas y divergentes realidades que emanan de una determinada situación, evento o realidad.

Además, quisiera mencionar los matices que MacKenzie y Bhatt (2018) añaden a este debate, sugiriendo que "La mierda es diferente de mentira y no tiene por qué socavar la confianza, especialmente cuando es evidente". Esto es de significativa relevancia en los tiempos contemporáneos, dados los movimientos populistas, las manifestaciones xenófobas y la denuncia de los derechos humanos, y la búsqueda de disminuir las "noticias" como "falsas" como principio básico que emana de algunos poderosos líderes del mundo occidental (Carr, 2020). Al mismo tiempo, es importante reconocer que los medios de comunicación tradicionales están igualmente anclados en los prejuicios y las trampas hegemónicas, pero les preocupa que la caravana de las "fake news" pretenda blanquear todo lo que pueda aportar dimensiones contrarias al debate (Feldman, Sol Hart y Milosevic, 2017). La complejidad de 
las interminables formas y formatos que surgen en los medios sociales hace que la curación de los medios, a veces en los feeds de noticias, pueda movilizar el apoyo popular y también diluirse y ahogarse al mismo tiempo.

\section{Considerando el entorno dentro de las redes sociales y las noticias falsas}

El planeta se enfrenta a cambios ambientales catastróficos, y los seres humanos están desempeñando sin duda un papel negativo en la aceleración de esta transformación (Carr, 2021). Podemos verlo, sentirlo, leer sobre él y, lo que es más importante, podemos hacer algo al respecto.

No comparto la idea de que la ciencia no tenga razón o que esto forme parte de un ciclo normal de 12.000 años o que tengamos que reflexionar más sobre esto antes de efectuar el cambio. Estamos perdiendo especies a un ritmo alarmante, las islas están siendo arrastradas hacia el océano, las enormes plataformas de hielo se están derritiendo, las temperaturas están aumentando, los bosques se reducen y el ser humano sigue pescando en exceso, lanzando bombas y manteniendo cantidades insostenibles de pobreza, todo lo cual sirve para degradar el medio ambiente en todas partes (Jandrić y Ford, 2020). Mucho se ha dicho y se dice sobre la legitimidad de la ciencia en la era de COVID-19, lo que ha alimentado un sin número algo sorprendente de personas en diversos contextos que se niegan a vacunarse, alegando que es parte de una conspiración, que no creen que lo necesiten o que su derecho a la libertad está por encima de la salud pública y otras posturas (Carr, 2020).

No estoy discutiendo aquí si el medio ambiente está cambiando, cuánto y por qué, sino que me interesa explorar el efecto del compromiso con las redes sociales, la participación ciudadana y la democracia en la forma en que se desarrolla el medio ambiente y el debate en torno a él (Hoechsmann, Thésée y Carr, 2021). Me interesan particularmente las dimensiones morales, éticas, epistemológicas, ideológicas, filosóficas, culturales, económicas y políticas de cómo se asume el medio ambiente en y a través de las redes sociales, y esto encaja en los debates sobre el significado, la existencia, la operacionalización, la influencia y la importancia de las noticias falsas (Carr, Cuervo y Daros, 2019; Carr, Daros, Cuervo y Thésée, 2020).

Al mismo tiempo que hay movilizaciones de masas, movimientos sociales, defensa e investigación en apoyo de la acción en relación con el medio ambiente, también somos conscientes de que los imperativos económicos neoliberales combinados con las posiciones políticas reaccionarias están cubriendo una parte importante del debate general y del compromiso educativo necesario. La presi- 
dencia de Trump, en particular, parece haberse complacido en refutar el cambio climático mundial, como lo demuestra su rechazo a los protocolos internacionales, la supresión de fondos para la investigación, el cambio de vocabulario y, en general, el avance de las políticas destinadas a la extracción de recursos no renovables (Carr, Molano, Rivas-Sanchez \& Thésée, 2018). Por ejemplo, Democracy Now (2019) presentó un informe titulado "Un negacionista del clima dirigirá el nuevo panel de Trump a pesar de que en su día comparó a los científicos del clima con los nazis", que recoge el espíritu de la hegemonía estadounidense en este ámbito.

Negar el cambio climático es un gran negocio. Nuccitielli (2018) documenta cómo el sector de las radios populistas de derecha de Estados Unidos, que tienen mucho peso y dólares, ha infestado el debate, siguiendo las acciones de un negador del clima particularmente vociferante.

La estrategia de Morano consiste en criticar dos de los estudios que han encontrado un consenso de expertos del 97\%. Uno de ellos, publicado en 2009 por Peter Doran y Maggie Zimmerman, encuestó a 3.146 científicos de la Tierra, pero menos de 80 de los participantes publicaban activamente investigaciones sobre el clima (y, por tanto, eran expertos en la materia). Básicamente, la crítica es que el tamaño de la muestra del estudio era demasiado pequeño para hacer una afirmación concluyente sobre el nivel de consenso de los expertos.

Es un punto válido, excepto que este era solo uno de los muchos estudios de consenso sobre el clima. De hecho, los autores de siete estudios de consenso distintos que utilizan una variedad de enfoques (algunos con tamaños de muestra muy grandes) se unieron en 2016 para publicar un documento que concluye que el consenso de los expertos sobre el calentamiento global causado por el hombre es de entre el 90 y el $100 \%$. Por lo tanto, esta crítica pierde validez, cuando se considera toda la investigación de consenso disponible.

La conexión con Facebook se ha planteado ampliamente en los círculos de las redes sociales. Nuccitielli (2018) señala que el video producido por el "negador del clima" en cuestión ha sido visto y, en consecuencia, compartidos en gran número (el recuento de cinco millones proporcionado por Facebook puede ser cuestionado por cuestiones de tiempo), difundiendo así información errónea que alterará las percepciones y el debate.

Blythe, Daigle y Baird (2019) sostienen que las palabras importan en lo que respecta al enfoque de su investigación sobre el medio ambiente al examinar "la resiliencia, la sostenibilidad y la transformación":

¿Recuerdan el "hermoso carbón limpio"? La administración Trump utilizó el término como la columna vertebral para el desarrollo continuo de la industria de 
los combustibles fósiles. Al mismo tiempo, sistemáticamente eliminó las palabras "cambio climático" de los sitios web federales, una medida destinada a socavar la acción climática.

Señalan hábilmente que identificar, dar forma y controlar el lenguaje, las metáforas, las imágenes y el debate, en este caso sobre el medio ambiente, tendrá importantes repercusiones en las formas políticas, económicas y socioculturales de abordar las cuestiones y preocupaciones medioambientales.

Nadie es dueño del significado de las palabras, sin embargo, es esencial tener en cuenta que el lenguaje nunca es neutral: transmite y moldea valores, actitudes e intenciones. Este poder sutil es especialmente preocupante ahora que los titulares falsos y politizados tienden a compartirse con más frecuencia que los reales.

Las palabras que utilizamos (y los significados que les atribuimos) se convierten en verdades públicas que establecen el contexto de las políticas, la financiación y las intervenciones medioambientales.

Smith (2018) destaca un artículo de 2018 en el Sunday Times, por ejemplo, que analizó indebidamente los resultados de un estudio de investigación que se había publicado en una revista académica en relación con la posible erupción de un volcán en Islandia. Estas informaciones erróneas, una vez publicadas por fuentes supuestamente creíbles y fiables, pueden (y probablemente lo harán) ser vertidas sin cesar, alcanzando y afectando a la conciencia pública antes de ser corregidas.

Una información inexacta significará, por supuesto, que la gente tendrá menos fe en los científicos y en las fuentes de noticias la próxima vez, aunque puede tener efectos inmediatos. En julio de 2018, el New York Times informó de cómo la cobertura exagerada de la erupción del Kilauea en curso en Hawái condujo (sic) a una percepción de riesgo enormemente inflada que vio disminuir las reservas turísticas, lo que a su vez provocó la pérdida de ingresos y el temor a la pérdida de empleos. En el peor de los casos, la mala información puede hacer que la gente ignore las órdenes de evacuación.

Lo que se omite, se minimiza y/o se ofusca en los informes, debates y cobertura de los medios de comunicación normativos saca a la luz la división entre ricos y pobres, entre el Norte y el Sur, entre poderosos y sin poder. Por ejemplo, en su informe Sufriendo en silencio: Las 10 crisis humanitarias menos difundidas en 2018, Care International (2019) destaca la siguiente relación dicotómica de varios niveles:

El mundo está marcado por la violencia y las catástrofes. El cambio climático provocado por las emisiones de combustibles fósiles golpea con más fuerza cada día que pasa. Sin embargo, algunas crisis reciben menos cobertura mediática que 
otras. El desplazamiento en la República Democrática del Congo rivaliza con el de Siria, pero ha recibido mucha menos atención. En la República Centroafricana se ha instalado una hambruna generalizada, que ha pasado prácticamente desapercibida. Y mientras que el catastrófico terremoto de 2010 en Haití ocupó los titulares, la crisis alimentaria de 2018 apenas fue noticia internacional. (p. 4)

La negligencia sistemática, dentro de los círculos políticos normativos, de conectar el cambio climático con las desigualdades sociales, la guerra y los conflictos, la migración masiva, el empobrecimiento y las experiencias vitales significativamente alteradas con nuestra propia agencia y capacidad de conciliar en qué se basa nuestra propia existencia material se juega a través de los medios de comunicación. Sin embargo, ¿quiénes son la normatividad, los medios de comunicación dominantes, las élites y los poderosos? ¿Y realmente tienen tanto poder e influencia como algunos creen que tienen? ¿No se trata de democratizar los espacios, las voces, las plataformas y los contenidos en las redes sociales?

La manera en que los medios de comunicación tradicionales y las redes sociales se relacionan con el medio ambiente tendrá, sin duda, un efecto significativo en las acciones que se lleven a cabo, ya que algunos estudios subrayan "que enmarcar el cambio climático como un problema de salud pública y no como una cuestión medioambiental es uno de los elementos que ayudaría a aumentar la participación del público en el compromiso con el cambio climático" (Depoux, Hémono, PuigMalet, Pédron, y Flahault, 2017, p. 1) confirmaron este hallazgo/recomendación a través de un amplio estudio de análisis de medios de comunicación utilizando datos del periódico Le Monde y de Twitter.

Del mismo modo, Wagner y Payne (2017, p. 1), en su estudio de tres de los periódicos más importantes de Irlanda"argumentan que los periódicos irlandeses no informan sobre el cambio climático de forma objetiva e imparcial e ilustran cómo a través de los actos de fijación de la agenda, el marco de las noticias y en cómo construyen el discurso público presentan el tema de forma ideológica estrecha" $y$, en consecuencia, "producen y reproducen una estrecha visión ideológica del mundo que es articulada, compartida y propagada por las élites políticas y económicas de Irlanda".

Además, en un amplio estudio internacional de 41 países y 113 periódicos nacionales, Barkemeyer, Figge, Hoepner, Holt, Kraak and Yu (2017, p. 18), destacan que "una serie de factores contextuales tienen una influencia significativa (positiva o negativa) que impactan en los niveles relativos de cobertura mediática en los países analizados" y que "los factores que impulsan la cobertura mediática no se limitan a la exposición de un país al cambio climático o a sus esfuerzos de mitigación. En 
cambio, el contexto más amplio en el que se desarrollan las políticas climáticas y la producción de los medios de comunicación desempeña un papel importante en particular en la gobernanza efectiva".

En esta línea, otro estudio de Feldman, Sol Hart y Milosevic (2015) analizó varios periódicos estadounidenses en el periodo 2006-2011 en relación con la "amenaza del cambio climático y la eficacia de las acciones para afrontarlo" (p. 481), concluyendo que, en general, las fuentes de noticias cubiertas no discutieron de manera consistente, sistemática o crítica el emparejamiento del impacto con lo que se podría hacer en relación con el cambio climático. Coincidimos con Carvalho, van Wessel y Maeseele (2016) en que las comunicaciones relacionadas con el cambio climático deben dirigirse directamente a la participación política, y es aquí donde los medios sociales tienen potencial para "democratizar" el debate, a pesar de todas las advertencias que ya hemos presentado.

Carmichael y Brulle (2016), en su revisión de 74 encuestas sobre el cambio climático en los EE.UU. para el período 2002-2013, encontraron que hay varios factores que pueden hacer saltar las alarmas relacionadas con la preocupación de los impactos ambientales.

Los principales factores que influyen en los niveles de preocupación pública por el cambio climático pueden agruparse en tres áreas. En primer lugar, ... la cobertura mediática del cambio climático influye directamente en el nivel de preocupación de las personas: cuanto mayor sea la cantidad de cobertura mediática del cambio climático, mayor será el nivel de preocupación de las personas ... La importancia que los medios de comunicación asignan a la cobertura del cambio climático se traduce en la importancia que las personas otorgan a esta cuestión. En segundo lugar, en una sociedad con un "espacio de emisión" limitado sobre el desempleo, la prosperidad económica y la participación en guerras que está compitiendo con la preocupación del cambio climático.

Sin embargo, el factor más importante que influye en la opinión pública sobre el cambio climático es la batalla partidista que las élites hacen sobre el tema. Los efectos más fuertes sobre la preocupación de la opinión pública están en función del aumento de la atención al Congreso sobre el cambio climático, que a su vez influye en la cobertura de los medios de comunicación y aumenta la preocupación por el cambio climático. De esta manera, este hallazgo mejora en gran medida nuestra comprensión de los factores que influyen en la preocupación que tiene el público por el cambio climático. (p. 16)

Pearce, Grundmann, Hulme, Raman, Hadley Kershaw y Tsouvalis (2017) se suman a esto sugiriendo que "los repetidos esfuerzos por apuntalar el consenso científico 
sobre las afirmaciones minimalistas como, "los seres humanos causan el calentamiento global" son distracciones de las cuestiones más urgentes del conocimiento, los valores, la elaboración de políticas y el compromiso público. Sostenemos que los investigadores que se preocupan por la relación entre el conocimiento y la política deberían invertir sus esfuerzos en estas áreas en lugar de en ejercicios de cuantificación del consenso sobre afirmaciones muy precisas de hechos científicos “ (p. 728). Estamos de acuerdo con Blue (2015) en que el debate y la deliberación pública en relación con el cambio climático necesitan una participación sólida de las ciencias sociales, además de las ciencias naturales, que podría ayudar a presentar "cuestiones alternativas, temas desatendidos, perspectivas marginadas y posibilidades diferentes que podrían ganar tracción a efectos políticos" (p. 1).

Más directamente en relación con las redes sociales, Fernández, Piccolo, Maynard, Wippoo, Meili y Alani (2016) emplearon la teoría de las 5 puertas del cambio de comportamiento (1. Deseabilidad, 2. Contexto propicio, 3. Puede hacer, 4. Buzz y 5. Invitación) al examinar las campañas sobre el cambio climático a través de Facebook y Twitter. Destacaron las motivaciones, la complejidad y los matices de la investigación sobre el uso, el compromiso y la influencia de los medios sociales en relación con el cambio climático. Es importante señalar que las campañas proambientales se beneficiarían de un mayor compromiso con las teorías del cambio de comportamiento a nivel de la organización y del individuo

Veltri y Atanasova (2015), a partir de los datos de los usuarios de Twitter, en su estudio sobre el cambio climático se revela el protagonismo de las fuentes mediáticas tradicionales que lideran e influyen en la ecología mediática y el intercambio de información a través de esta red.

\begin{abstract}
El análisis de la ecología de los medios de comunicación revela una dependencia de las fuentes profesionales de información, como los periódicos y la radiodifusión pública. En este caso, los usuarios de Twitter recurrieron más a las fuentes tradicionales donde hay poca diversidad ecológica de fuentes de la World Wide Web. Por lo tanto, los resultados relacionados con el grado de sofisticación del discurso pueden reflejar simplemente el discurso de los medios de comunicación tradicionales. Sin embargo, en Twitter, las fuentes tradicionales fueron seleccionadas con notable sofisticación, y los usuarios se comportaron más bien como "curadores". [...] En cualquier caso, la falta de diversidad de fuentes es notable y contrasta con las expectativas comunes sobre la diversidad de canales de comunicación en línea. (p. 13)
\end{abstract}

En cuanto a la cuestión de cómo la gente puede utilizar los medios sociales de forma diferente a los medios tradicionales, cambiando así entre la Democracia/Medios 
1.0 y la Democracia/Medios 2.0. Por ejemplo, Spartz, Yi-Fan Su, Griffin, Brossard y Dunwoody (2017) experimentaron con estudiantes universitarios de Estados Unidos en relación con Youtube y la importancia de las pistas, como el número de visitas que ha recibido un vídeo.

[...] las plataformas de las redes sociales ofrecen un terreno fértil para este tipo de indicios, quizás incluso más que los medios de comunicación convencionales del pasado. No es que las fuentes de los medios de comunicación tradicionales no ofrecieran o no ofrezcan señales sociales, sino que un elemento inherente a la Web 2.0 es la sociabilidad y la conexión en red por encima de las capacidades de los medios tradicionales. Los canales sociales como YouTube, Facebook y Twitter conducen ciertamente a los usuarios a las noticias y otra información objetiva, igual que los medios de comunicación tradicionales, pero los canales sociales también presentan una fuerte superposición de información personal que está saturada de potencial normativo - una distinción de los formatos mediáticos del pasado. Incluso los enlaces ostensiblemente neutrales a las noticias de YouTube, Las normas sociales y el cambio climático. Las historias en un tweet contienen el mensaje normativo de que alguien a quien "sigues", probablemente porque respetas a esa persona, piensa que los demás deberían prestar atención al tema. (pp. 11-12)

En resumen, el debate sobre el cambio climático avanza y retrocede simultáneamente, y está siendo engatusado por noticias falsas, engañosas y tergiversadas y masajeado en varios rincones de la política formal, así como en la conciencia de la sociedad civil. Además, está sirviendo para reflejar muchas de las rupturas y epifanías propias de los medios sociales, las redes sociales y la participación ciudadana. Al mismo tiempo, no se puede culpar a los medios sociales de forma generalizada por la falta de acción transformadora y crítica en relación con el medio ambiente. Por el contrario, gran parte de la movilización ha sido estimulada por la organización, las comunicaciones y la solidaridad que se han generado a través de grupos y movimientos que utilizan eficazmente las comunicaciones digitales y de Internet, así como los medios sociales.

\section{Discusión}

Abordar el cambio ambiental, o más bien la catástrofe ambiental, requiere un compromiso político que vaya más allá de las promesas formales, los compromisos, los acuerdos, las metas, los objetivos y los planes que rara vez se cumplen. Es claro que las acciones formales y los recursos son extremadamente importantes, 
y los eventos anuales de la COP que sirven como centros significativos de diálogo y compromiso que podría influir mucho y cambiar el debate. Al mismo tiempo, no podemos esperar a que los sistemas políticos neoliberales y bélicos se transformen en activistas medioambientales, que denuncien el extractivismo, las prospecciones petrolíferas, el expansionismo militarizado y el saqueo de los océanos (Carr, Molano, Rivas-Sanchez y Thésée, 2018).

Reconocer el lugar y el papel de los movimientos de solidaridad que organizan, informan, educan y persuaden a las comunidades, los países y el mundo para que actúen es un pilar fundamental para transformar el statu quo. La ecopedagogía implica la combinación de un compromiso consciente con una preocupación por la justicia social, los pueblos originarios, todas las especies y consideraciones transformadoras de las realidades y condiciones sociopolíticas y económicas que nos explotan, disminuyen, marginan o destruyen a las personas y comunidades (Jandrić y Ford, 2020). Los medios sociales se entrelazan con estos procesos, sistemas, planes y organización.

El medio ambiente está enmarañado con la agencia humana y las existencias humanas, y el movimiento en los ámbitos de las desigualdades sociales, la pobreza, la autocracia y la calidad de vida, para todos, es fundamental que alteremos el curso de colisión que tenemos ante nosotros. Además, es fundamental la reconciliación con los pueblos originarios y el respeto a sus reivindicaciones territoriales, también es necesario que replanteemos la democracia más allá de las elecciones para que incluyamos un compromiso sólido y crítico. En última instancia, la alfabetización política y mediática depende de la educación a nivel formal, informal y no formal, y la educación transformadora orientada a la ecopedagogía sólo puede tener lugar cuando se acepten las condiciones y experiencias reales y materiales tal y como son. El mundo es extremadamente discriminatorio, y el racismo, el sexismo, el clasismo y otras formas de marginación deben incluirse en este debate. El racismo ambiental implica al medio ambiente $\mathrm{y}$ al racismo, y reconsidera a la desigualdad de manera más amplia lo que puede permitirnos ir más allá de las preocupaciones locales, aunque trabajar a nivel local también es esencial (Carr y Thésée, 2019).

Los medios sociales son la estructura que enmarca todos estos problemas y procesos arraigados y cargados de poder. Brady, Wills, Jost, Tucker and Van Bavel (2017) destacan el papel de la emoción en la comunicación de los medios sociales y las redes en lo que respecta a la divulgación y difusión de mensajes en relación con temas de gran carga política, como el cambio climático. La forma en que dialogamos entre nosotros es muy importante, y ninguno de los grandes problemas casi insuperables a los que se ha aludido anteriormente pueden imaginarse sin 
un diálogo y una democracia significativos, críticos y deliberativos. Necesitamos liderazgo, pero también necesitamos participación, compromiso e innovación.

Con respecto a la democracia, sería importante reimaginarla no solamente en las instituciones, los procesos, sistemas y recursos tradicionalmente vinculados al tema, pero, especialmente, en la educación. La democracia sin la educación crítica, involucrada y pertinente es difícil de conceptualizar. Sería importante repensar en todos los componentes de la experiencia educativa, incluyendo la pedagogía, el currículo, el liderazgo, la cultura institucional, la epistemología, las políticas educativas y la experiencia formal, informal y nonformal (Carr y Thésée, 2019). Necesitamos también reconsiderar cómo hacer y vivir la democracia en, con, a través y vinculando a la educación. Problematizar los objetivos de la educación, enfrentar al neoliberalismo y las desigualdades sociales y cultivar activamente la agencia, la praxis y el involucramiento exige un cambio de paradigma. La conexión, entonces, con las redes sociales me parece fundamental aquí. Además, la centralidad del medio ambiente no puede ser abordado, creo, sin esta trinidad de conceptos (democracia, redes sociales, medio ambiente) y, claro, la educación transformadora para facilitar, apoyar e instrumentalizar el diálogo necesario para crear la solidaridad social.

No es posible diseccionar fácilmente todas las demás cuestiones presentadas anteriormente, no obstante, me interesa la configuración, el marco teórico, el posicionamiento y la presentación de estos temas, y de los muchos otros que no reciben tanta exposición. La falta de contexto histórico, político y económico, combinada con la propensión a no aferrarse a la "investigación" y a la pluralidad de visiones, perspectivas y experiencias, parece ser un rasgo predominante de cómo se cristalizan muchas de estas historias. Cada uno de los ejemplos aludidos contiene un conjunto de circunstancias y marcos en evolución, así como preguntas, y soy consciente de cómo algunos segmentos de los medios de comunicación social pueden proporcionar narrativas diferentes que, en consecuencia, pueden remodelar la historia "oficial". El medio ambiente y el clima seguirán siendo preocupaciones centrales para toda la humanidad, y la forma en que nos relacionemos unos con otros, incluso en los medios sociales y a través de ellos, lo que será decisivo para saber dónde acabaremos. 


\section{Bibliografía}

Barkemeyer, R., Figge, F., Hoepner, A., Holt, D., Kraak, J. M. y Yu, P. S. (2017). Media coverage of climate change: An international comparison. Environment and Planning C: Politics and Space. DOI: 10.1177/0263774X16680818

Blue, G. (2015). Framing Climate Change for Public Deliberation: What Role for Interpretive Social Sciences and Humanities? Journal of Environmental Policy \& Planning. DOI: 10.1080/1523908X.2015.1053107

Blythe, J., Daigle, C. y Baird, J. (2019). The meaning of environmental words matters in the age of 'fake news', The Conversation (January 10). http://theconversation.com/ the-meaning-of-environmental-words-matters-in-the-age-of-fake-news-106050

Brady, W. K., Wills, J. A., Jost, J. T., Tucker, J. A. \& Van Bavel, J. J. (2017). Moral contagion in social networks. Proceedings of the National Academy of Sciences, 114 (28), 73137318. DOI:10.1073/pnas.1618923114

Care International. (2019). Suffering In Silence The 10 most under-reported humanitarian crises of 2018. https://care.ca/wp-content/uploads/2019/02/02072019_Report_ Suffering-In-Silence-2018_ENG_WEB_final.pdf

Carmichael, J. y Brulle, R. (2016). Elite cues, media coverage, and public concern: an integrated path analysis of public opinion on climate change, 2001-2013, Environmental Politics. DOI: 10.1080/09644016.2016.1263433

Carr, P. (2021). El paciente está de rodillas: ¿Está la democracia en un estado pandémico? In Aparici, R. \& Martinez-Pérez, J. (Ed.), El algoritmo de la incertidumbre. Barcelona: Editorial Gedisa.

Carr, P. (2020). Shooting yourself first in the foot, then in the head: Normative democracy is suffocating, and then the Coronavirus came to light. Postdigital Science and Education, 1-19. https://doi.org/10.1007/s42438-020-00142-3

Carr, P., Cuervo, S. \& Daros, M. (2019). Citizen engagement in the contemporary era of fake news: Hegemonic distraction or control of the social media context? Postdigital Science and Education, 1-22. https://link.springer.com/article/10.1007/s42438019-00052-z\#citeas

Carr, P., Daros, M., Cuervo, S., y Thésée, G. (2020). Social media and the quest for democracy: Faking the re-awakening?. In Trifonis, Peter (ed.), Handbook of Theory and Research in Cultural Studies and Education (1-24). New York: Springer. https://link.springer. com/referenceworkentry/10.1007\%2F978-3-030-01426-1_31-1\#citeas

Carr, P., Hoechsmann, M. y Thésée, G. (eds.). (2018). Democracy 2.0: Media, Political Literacy and Critical Engagement. Rotterdam: Brill/Sense 
Carr, P., Molano, N., Rivas-Sanchez, E., y Thésée, G.. (2018). Pedagogías contra el despojo: Principios de una eco/demopedagogía transformativa como vehículo para la justicia social y ambiental en América Latina, Revista Internacional de Educación para la Justicia Social, 7(1), 45-69. https://revistas.uam.es/riejs/article/view/9580

Carr, P. y Thésée, G. (2019). "It's not education that scares me, it's the educators...": Is there still hope for democracy in education, and education for democracy? Gorham, ME: Myers Education Press.

Carvalho, A., Van Wessel, M. y P. Maeseele (2016). Communication practices and political engagement with climate change: A research agenda. Environmental Communication, http://dx.doi.org/10.1080/17524032.2016.1241815

De Coninck, D. et al. (2021). Beliefs in Conspiracy Theories and Misinformation About COVID-19: Comparative Perspectives on the Role of Anxiety, Depression and Exposure to and Trust in Information Sources. Front. Psychology (16 April). https:// doi.org/10.3389/fpsyg.2021.646394

Democracy Now. (2019). Climate Denier to Head New Trump Panel Despite Once Comparing Climate Scientists to Nazis (February 21). https://www.democracynow. org/2019/2/21/climate_denier_to_head_new_trump?fbclid=IWAR1kLfa-gG4y2PPQlyKOSUDgZd62t3FKa0eu6d2dZVpR3kHtWPEkZQbKLc

Depoux, A., Hémono, M., Puig-Malet, S., Pédron, R. y Flahault, A. (2017). Communicating climate change and health in the media. Public Health Reviews, 38(7), 1-4.

Feldman, L., Hart, P. y Milosevic, T. (2017). Polarizing news? Representations of threat and efficacy in leading US newspapers' coverage of climate change. Public Understanding of Science, 26(4), 481-497. https://doi.org/10.1177/0963662515595348

Fernandez, M., Piccolo, L., Maynard, D., Wippoo, M., Meili, C. y Alani, H. (2016). Talking Climate Change via Social Media: Communication, Engagement and Behaviour. In: WebSci '16 Proceedings of the 8th ACM Conference on Web Science, ACM, New York, USA, pp. 85-94.

Hoechsmann, M., Carr, P. \& Thésée, G. (2021). The Struggle Over Meaning in A World in Crisis. In Hoechsmann, M., Thésée, G. \& Carr, P. R. (eds.), Education for Democracy 2.0: Changing Frames of Media Literacy (pp. 1-10). Rotterdam: Brill/Sense Publishers.

Hoechsmann, M., Thésée, G. \& Carr, P. (eds.). (2021). Education for Democracy 2.0: Changing Frames of Media Literacy. Rotterdam: Brill/Sense.

Jandrić, P. y Ford, D. (2020). Postdigital Ecopedagogies: Genealogies, Contradictions, and Possible Futures. Postdigit Sci Educ. https://doi.org/10.1007/s42438-020-00207-3

Keating, A. y Melis, G. (2017). Social media and youth political engagement: Preaching to the converted or providing a new voice for youth? The British Journal of Politics and International Relations, 19(4), 877-894. 
MacKenzie, A. y Bhatt, I. (2018). Lies, Bullshit and Fake News: Some Epistemological Concerns. Postdigital Science and Education, 1-5.

Nuccitielli, D. (2018). Facebook video spreads climate denial misinformation to 5 million users. The Guardian (July 22). https://www.theguardian.com/environment/ climate-consensus-97-per-cent/2018/jul/25/facebook-video-spreads-climatedenial-misinformation-to-5-million-users

Pearce, W., Grundmann, R., Hulme, M., Raman, S., Hadley, E. y Tsouvalis, J. (2017) Beyond Counting Climate Consensus. Environmental Communication, 11:6, 723-730. DOI: 10.1080/17524032.2017.1333965

Smith, K. (2018). What an Icelandic volcano tells us about fake news. World Economic Forum (in collaboration with The Conversation) (October 17). https://www.weforum.org/ agenda/2018/10/what-an-icelandic-volcano-tells-us-about-fake-news

Spartz, J., Yi-Fan, L., Griffin, R., Brossard, D. y Dunwoody, S. (2017). YouTube, Social Norms and Perceived Salience of Climate Change in the American Mind. Environmental Communication, 11(1), 1-16. DOI:10.1080/17524032.2015.1047887

Sun, Y., Wang, N., Shen, X. y Zhang, J. (2015). Location information disclosure in locationbased social network services: Privacy calculus, benefit structure, and gender differences. Computers in Human Behavior, 52, 278-292.

Sutton, K. (2018). Facebook's Terrible, Horrible, No Good, Very Bad Year: And you thought you had a rough 2018?. Adweek (December 26). https://www.adweek.com/digital/ facebooks-terrible-horrible-no-good-very-bad-year/

Thésée, G. y Carr, P. (2020). Paulo Freire, critical pedagogy and the quest for"Transformative and Emancipatory Education (TEE)". In Steinberg, S. \& Down, B. (eds.), The SAGE Handbook of Critical Pedagogies (pp. 67-74). London: Sage Publications.

University of West Florida, University Libraries (2019). EVR 2001: Introduction to Environmental Science: What kinds of fake news exist? https://libguides.uwf.edu/c. php?g=436278\&p=4297847

Veltri, G. y Atanasova, D. (2015). Climate change on Twitter: Content, media ecology and information sharing bahviour. Public Understanding of Science, 1-17. DOI: $10.1177 / 0963662515613702$

Wagner, P. y Payne, D. (2017). Trends, frames and discourse networks: analysing the coverage of climate change in Irish newspapers, Irish Journal of Sociology, 25(1) 5-28.

Wong, J. y Morris, S. (2018). It's complicated: Facebook's terrible 2018. The Guardian (December 24). https://www.theguardian.com/technology/ng-interactive/2018/dec/24/ facebook-2018-timeline-year-in-review-privacy-scandals 